\title{
The Effects of Intranasal Dexmedetomidine Premedication on Sedation and Stress of Tracheal Intubation in Patients Undergone Gynecological Laparoscopic Surgery
}

\author{
Xiaoxia Gu, Xiu Juan Tan, Ying-Xia Guo, Shuyun Cai, Jinxian Chen and Liangqing Zhang* \\ Department of Anesthesiology, China
}

*Corresponding author: Liang Qing Zhang, Department of Anesthesiology, China

\section{ARTICLE INFO}

Received: 幽 October 04, 2019

Published: 幽 October 11, 2019

Citation: Xiaoxia Gu, Xiu Juan T, Ying-Xia G, Shuyun C, Jinxian C, Liangqing Z. The Effects of Intranasal Dexmedetomidine Premedication on Sedation and Stress of Tracheal Intubation in Patients Undergone Gynecological Laparoscopic Surgery. Biomed J Sci \& Tech Res 21(5)-2019. BJSTR. MS.ID.003677.

Keywords: Dexmedetomidine; Intranasal; Premedication; Sedation; Stress; Tracheal Intubation

Abbreviations: Dex: Dexmedetomidine; $\mathrm{SPO}_{2}$ : Oxygen Saturation; BIS: Bispectral Index; COR: Circulating Cortisol; E: Epinephrine; N: Norepinephrine; BS: Blood Sugar

\begin{abstract}
Background: Dexmedetomidine (Dex) is a highly potent, highly selective adrenergic alpha 2 receptor agonist that has its unique advantages over midazolam: "wakeable" sedative state and inhibiting sympathetic activity. This study is a new attempt for premedication to determine whether intranasal Dex offered perioperative sedation and better attenuation of stress responses to tracheal intubation in patients undergone gynecological laparoscopic surgery.
\end{abstract}

Methods: forty cases were randomly allocated according to computer generated randomization to receive either Group D(Intranasal administrated to each naris as drops dexmedetomidine $1.0 \mathrm{ug} / \mathrm{kg} 30 \mathrm{~min}$ before induction of anesthesia, $\mathrm{n}=20$ ) or Group C(placebo, Intranasal administrated the same volume of $0.9 \%$ saline, $n=20$ ). Haemodynamic parameters, oxygen saturation $\left(\mathrm{SpO}_{2}\right)$, Bispectral Index (BIS), the Ramsay sedation score and serum Concentrations of Cortisol (COR), Adrenaline (E) and Norepinephrine (NE) were measured by ELISA.

Result: In the group D, BIS values were significantly lower and Ramsay sedation scores was higher before surgery $(\mathrm{P}<0.05)$, And heart rate, Systolic blood pressure and rate pressure product was significantly lower than group $C$ before surgery and at tracheal intubation $(\mathrm{P}<0.05)$. There were no differences between two groups in $\mathrm{SpO} 2$ at before and after the medication $(\mathrm{P}<0.05)$. In the group $\mathrm{C}$, plasma concentrations of Cor, $\mathrm{E}$ and $\mathrm{BS}$ were increased significantly $(\mathrm{P}<0.05)$.

Conclusion: intranasal administration of dexmedetomidine $(1 \mathrm{ug} / \mathrm{kg}) 30 \mathrm{~min}$ before operation can be effectively applied sedation and alleviate stress response to tracheal intubation of patients, it is as a safe and effective preoperative medication.

Question: Is intranasal Dex offered perioperative sedation and better attenuation of stress responses to tracheal intubation in patients undergone gynecological laparoscopic surgery?

Findings: In the Dex group, BIS values were significantly lower and Ramsay sedation scores was higher before surgery.

Meaning: Intranasal administration of Dex $(1 \mathrm{ug} / \mathrm{kg}) 30 \mathrm{~min}$ before operation is a safe and effective preoperative medication.

\section{Introduction}

Studies have shown that patients have different degrees of anxiety before surgery, the incidence is generally about $60 \%$, up

to $80 \%$ [1]. When patients have excessive nervous and anxious emotional reaction to surgery before surgery, it will directly affect 
the smooth operation and the recovery of the body after surgery [2]. Therefore, the main purpose of preoperative medication is to patient sedates and relieves anxiety. Dex is a highly effective and highly selective adrenergic alpha 2 receptor agonist with sedative, analgesic and better attenuation of stress responses properties that produces less respiratory suppression [3]. It is a new attempt in preoperative medication. Compared with midazolam, dexmedetomidine has its unique advantages: it is a new sedative and analgesic drug because of its unique "wake-up" sedative state and inhibition of sympathetic activity [4,5]. With the emergence of comfortable medical treatment and humanized anesthesia, non-invasive administration gradually replaces intramuscular administration. Nasal administration does not pass through the first-pass effect of the liver. It is small and non-invasive, and patients feel comfortable. It has attracted more and more attention and love at home and abroad. Intranasal Dex was found to produce more satisfactory premedication sedation than oral midazolam in children with burns [6]. This study was designed to observe the effects of preoperative intranasal Dex on sedation, anti-anxiety and hemodynamics in patients undergoing gynecological laparoscopic surgery.

\section{Materials and Methods}

All patients participating in the trial were approved by the ethics committee of the Affiliated Hospital of Guangdong Medical University and signed the informed consent before operation. The trial was registered prior to patient enrollment at clinicaltrials. gov (Effects of dexmedetomidine combined with parecoxib sodium in patients undergoing endoscopic nasal surgery rapid rehabilitation and prognosis, Principal investigator: GU XiaoXia, Date of registration:16 January 2017). Trial's clinical trial registration number is ChiCTR-OPN-17010444. Forty gynecological laparoscopic patients aged 20-50 years with ASA grade I-II were selected. All patients were approved by the ethics committee of the hospital and signed the informed consent before operation. Exclusion criteria included anticipated difficult intubation, clinical history or electrocardiographic evidence of heart block, severe systemic diseases involving the renal and hepatic systems, ischaemic heart disease, pregnancy, known psychiatric illness, chronic sedative or analgesic use, nasal disease and intubation attempt lasting longer than 15 seconds.

All the patients were randomly allocated according to computer generated randomization to receive either Group D(Intranasal administrated to each naris as drops dexmedetomidine $1.0 \mathrm{ug} / \mathrm{kg}$ 30 min before induction of anesthesia, $n=20$ ) or Group C(placebo, Intranasal administrated the same volume of $0.9 \%$ saline, $\mathrm{n}=20$ ). One hour before surgery, patients were changed in SBP(Systolic blood pressure), HR(heart rate), rate pressure product (RPP), Sp02(oxygen saturation), BIS(bispectral index) and the Ramsay sedation score (RSS) (Table 1) were recorded at $5 \mathrm{~min}$ before the drug $\left(\mathrm{T}_{0}\right)$ then $10 \mathrm{~min}\left(\mathrm{~T}_{1}\right) 20 \mathrm{~min}\left(\mathrm{~T}_{2}\right), 30 \mathrm{~min}$ after the drug (T3). Intravenous infusion of Ringer's lactate solution $8-10 \mathrm{ml} /$ $\mathrm{kg}$, operated by a fixed skilled anesthesiologist, was induced after 3 minutes of denitrification and oxygen supply. Intravenous administration of propofol $2 \mathrm{mg} / \mathrm{kg}$, atracurium cis-phenylsulfonate $0.2 \mathrm{mg} / \mathrm{kg}$, and Sufen $0.5 \mathrm{ug} / \mathrm{kg}$ were given. At the same time, the flow rate of fresh oxygen inhaled by mask was set to $3 \mathrm{~L} / \mathrm{min}$, after the patient's consciousness disappeared. After muscle relaxation, endotracheal intubation was performed under laryngoscope. Tracheal tube selection "7.0-7.5". The surface is fully lubricated, requiring one-time intubation success, mechanical ventilation after intubation, set: VT 10ml kg-1, RR12 times per minute.Hemodynamic changes were recorded again before induction $\left(\mathrm{T}_{4}\right)$, before trachea cannula after induction $\left(\mathrm{T}_{5}\right), 1\left(\mathrm{~T}_{6}\right), 3\left(\mathrm{~T}_{7}\right)$ and $6\left(\mathrm{~T}_{8}\right)$ minutes after trachea cannula during anesthetic induction. Venous blood samples were collected to measure the levels of NE (norepinephrine), E(epinephrine), Cor(cortisol) by Enzyme-linked immunosorbent assay method and the BS (blood sugar) concentration by blood glucose meter at the time of $\mathrm{T}_{0}, \mathrm{~T}_{4}$ and $\mathrm{T}_{7}$.

Table 1: Ramsay sedation score.

\begin{tabular}{|c|c|}
\hline $\begin{array}{c}\text { Sedation } \\
\text { score }\end{array}$ & Status \\
\hline 1 & Anxious, agitated, restless \\
\hline 2 & Eyes open, co-operative, oriented, tranquil \\
\hline 3 & Responds (open eyes), only to command, light touch, \\
normal tone of voice
\end{tabular}

\section{Statistical and Analysis}

SPSS 18.0 statistical software (SPSS Institute, Chicago, IL, USA) was used for analysis. The measurement data of normal distribution are expressed as mean ( ). The comparison among groups is based on one-way ANOVA, while the comparison within groups is based on the ANOVA of repeated measurement design. Chi - square test was used to analyze the categorical data and for testing the association between the variables. Nonparametric tests (Wilcoxon signed rank tests [two - tailed]) were used whenever the mean value was less than two times the standard deviation. $\mathrm{P}<0.05$ showed statistical significance.

\section{Results}

All 40 patients completed the study. There was no significant difference in age, height, weight and ASA grade between the two groups ( $\mathrm{P}>0.05$ ) (Table 2). In group D the Ramsay scores were obviously higher and the BIS value were notably lower after intranasal administration of drug than group $\mathrm{C}(\mathrm{P}<0.05)$. While there were no differences in Ramsay score and BIS value in group $\mathrm{C}$ at all time points $(\mathrm{P}>0.05)$ (Table 3 ). There was no significant difference in SBP, DBP, HR before administration between the two groups $(P>0.05)$. HR values were statistically significantly lower in the group $\mathrm{D}$ at all time intervals compared to the group $\mathrm{C}$. There was a statistical significance in the SBP and RPP between groups before intubation and after intubation at the 1st, 3rd and 6th min 
$(\mathrm{P}<0.05)$. In the group $\mathrm{C}, \mathrm{HR}, \mathrm{SBP}$ and RPP increased significantly compared to baseline, starting from intubation and continuing until the end of the study. The group D had a better control of HR, SBP and RPP than the group C (Figure 1). There was no significant difference in NE, E, Cor and the BS concentration before administration between the two groups $(\mathrm{P}>0.05)$. In group $\mathrm{D}, \mathrm{NE}, \mathrm{E}$, Cor and the BS concentration decreased significantly compared to baseline and group $\mathrm{C}$ after intranasal administration of drug. While all aforementioned variables were significantly higher in group $\mathrm{C}$ compared with the group D after intubation $(\mathrm{P}<0.05)$ (Figure 2).

Table 2: Patient characteristics and preoperative data.

\begin{tabular}{|c|c|c|c|}
\hline Variable & Group D (n=20) & Group C (n=20) & P-value \\
\hline Age & $39.0(7.9)$ & $36.3(9.3)$ & 0.328 \\
\hline Body weight (kg) & $52.7(7.4)$ & $53.9(8.6)$ & 0.6389 \\
\hline Body height (cm) & $156.4(5.0)$ & $157.7(3.8)$ & 0.3628 \\
\hline ASA physical status ( I / I ) & $13 / 7$ & $10-0 c t$ & 0.337 \\
\hline
\end{tabular}

Note: Values are expressed as mean \pm standard deviation (SD).

Table 3: Patient characteristics and preoperative data.

\begin{tabular}{|c|c|c|c|}
\hline & Group D (n=20) & Group C (n=20) & P-value \\
\hline RSS T0 & $1.25(0.44)$ & $1.27(0.47)$ & 0.8902 \\
\hline T1 & $1.40(0.50)$ & $1.35(0.49)$ & 0.7389 \\
\hline T2 & $1.80(0.55)$ & $1.40(0.50)$ & 0.0162 \\
\hline T3 & $2.25(0.55)$ & $1.35(0.48)$ & 0 \\
\hline BIS T0 & $91.9(3.3)$ & $91.2(3.5)$ & 0.5152 \\
\hline T1 & $85.9(2.9)$ & $89.9(2.9)$ & 0.0001 \\
\hline T2 & $83.6(2.41)$ & $89.8(2.26)$ & 0 \\
\hline T3 & $80.2(2.8)$ & $89.1(2.25)$ & 0 \\
\hline
\end{tabular}

Note: RSS - Ramsay sedation score; T0 - Baseline; T1 - 10 min after drug; T2 - 20 min after drug; T3 - 30 min after drug. Values are expressed as mean \pm standard deviation (SD).
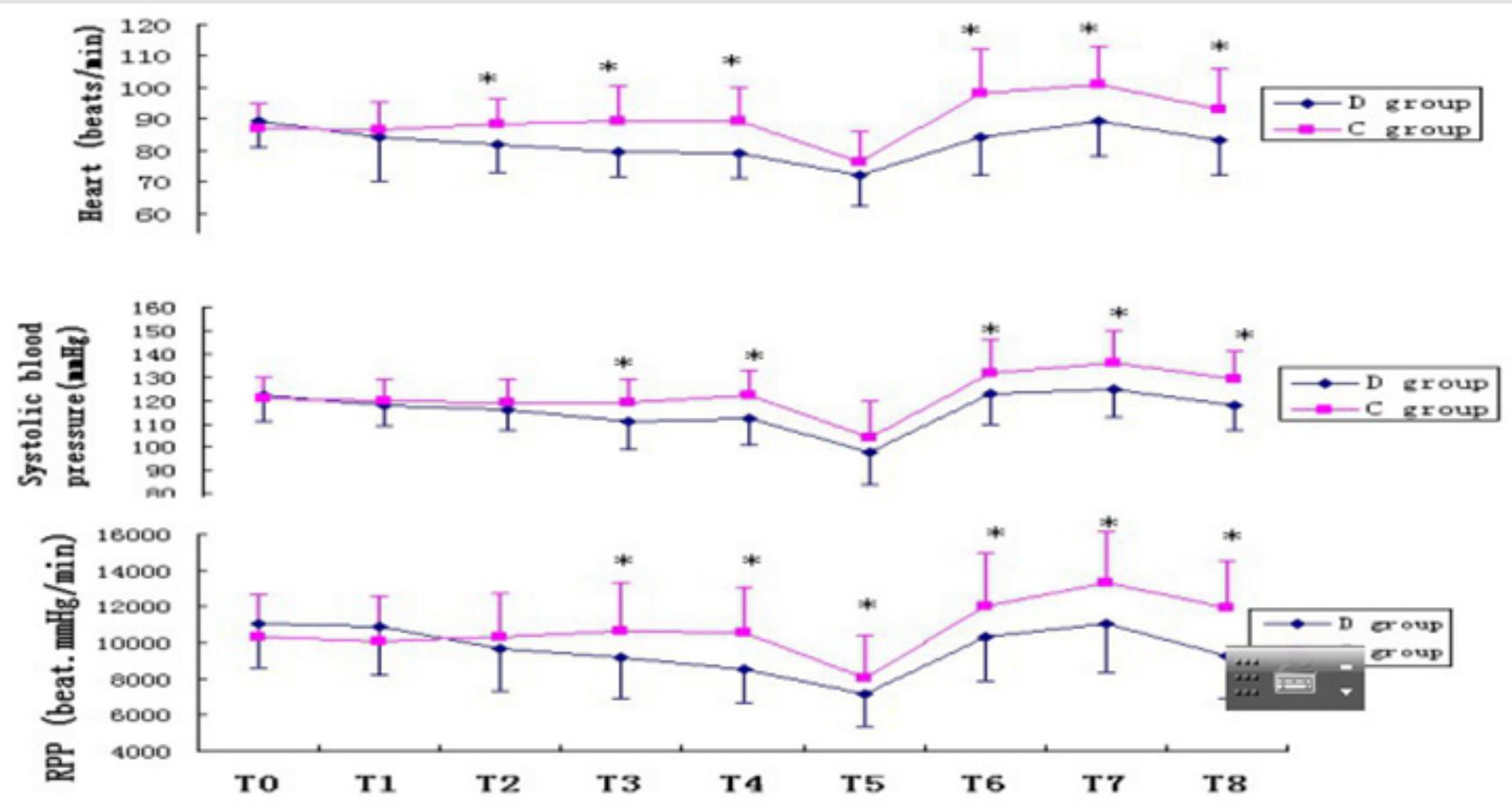

Figure 1: HR- Heart Rate; SBP- Systolic Blood Pressure; RPP- Rate Pressure Product, T0- Bseline; T1- 10min after drug; T220min after drug; T3- 30min after drug; T4- Before Intubation; T5- Before trachea cannula after induction; T6- First minute after Intubation; T7- Third minute after Intubation; T8- Sixth minute after Intubation; ${ }^{*}<0.05$ Compared with control group. 

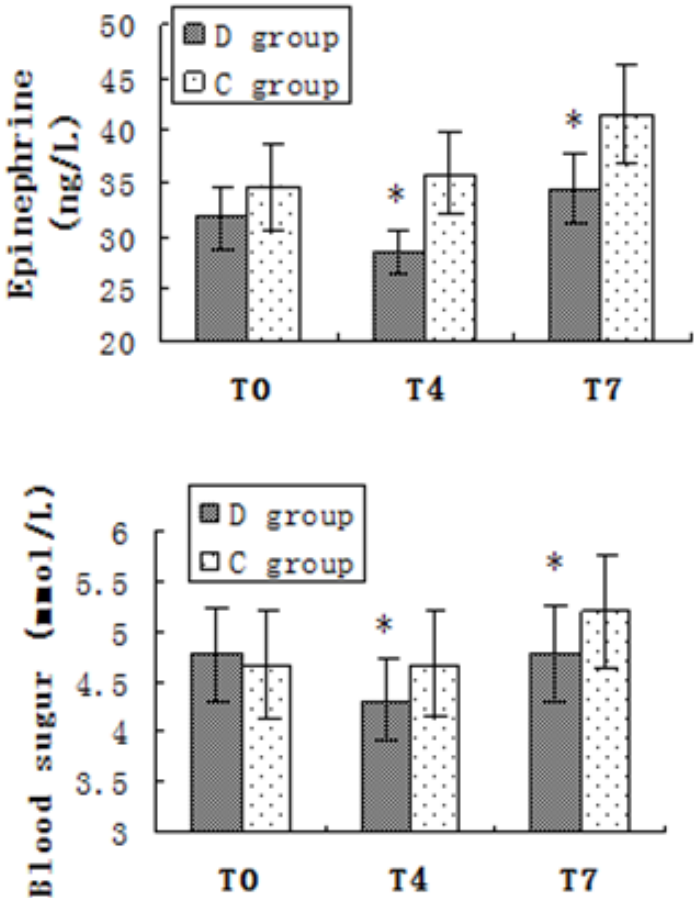
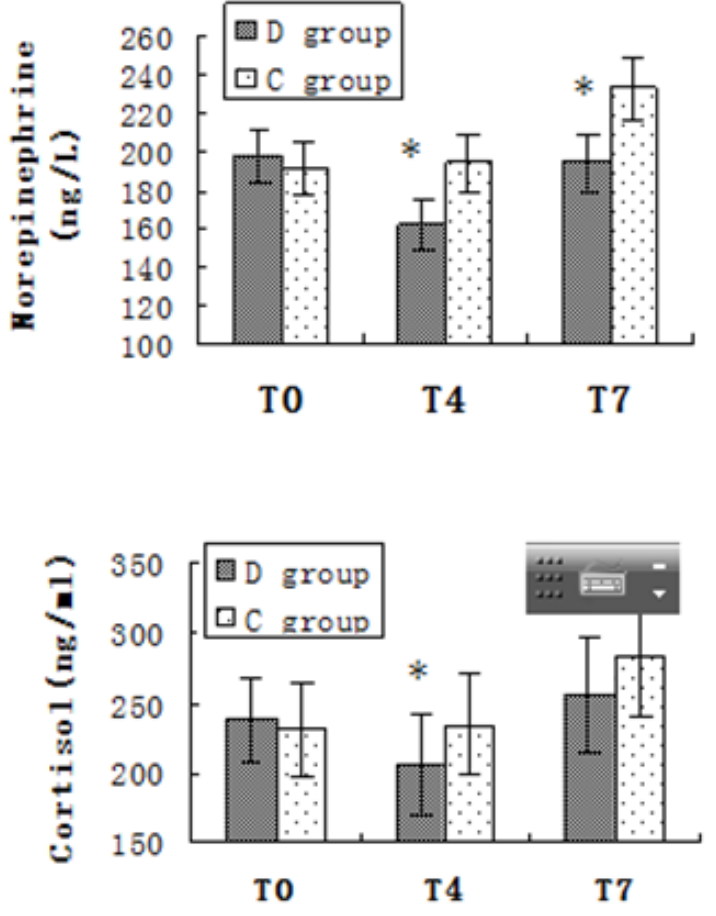

Figure 2: Plasma Epinephrine, norepinephrine, cortisol and blood sugar level. Columns are mean, and error bars are standard deviations. ${ }^{*} P<0.05$ Compared with control group. T0-Baseline; T4-Before intubation; T7- Third minute after intubation.

\section{Discussion}

The present study demonstrated that patients given intranasal Dex in gynecological laparoscopic surgery were more sedated perioperatively and had a better control of HR, SBP and RPP. Decreased NE, E, Cor and the BS concentration showed that the stress response was inhibited. With the advent of comfortable medical treatment and humanized anesthesia, non-invasive preoperative administration has great prospects of gradually replacing intramuscular administration. Nasal administration can quickly absorb blood through nasal mucosa to achieve systemic circulation. Without the first-pass effect of liver, it is small, noninvasive and comfortable for patients. Nasal drug delivery system has tremendous potential for development. It is considered to be the most feasible alternative to injections because of its rapid onset, remarkable efficacy and high patient compliance. Dex is a colorless and odorless isotonic solution, which has no stimulating effect on nasal mucosa, and its bioavailability is $82 \%$ [7].

Therefore, intranasal administration is a simple, convenient, comfortable and effective way of administration. The patient feels comfortable and the drug can reach the central nervous system directly through the surface blood vessels of nasal mucosa. The system avoids the first-pass effect of the liver [8,9]. Dex can exert analgesic effect by activating locus coeruleus in the brainstem of the alpha 2 receptor in the nervous system, as well as acting on the alpha 2 receptor in the presynaptic membrane and neuron post- synaptic membrane of the spinal dorsal foot, which produces a sedative effect similar to natural sleep $[10,11]$. During clinical anesthesia, it is easy to increase the depth of anesthesia, reduce the dosage of other anesthetics, and maintain more stable vital signs [12]. Studies have shown that [13]. Dex given $1 \mathrm{ug} / \mathrm{kg}$ before induction of anesthesia is prone to hypotension and sinus bradycardia, and the incidence is higher in healthy patients. There were also researchers [14] who used 0.25-0.5ug/kg dexmedetomidine without bi-directional changes in blood pressure. Yuen's study involved 18 healthy adult volunteers who were given Dex (1-1.5ug/kg) intranasally drip, the peak time of plasma was 60-150 minutes and the duration was 180 minutes [15].

While intranasal spray Dex produces sedation at 25-30min, duration $85 \mathrm{~min}$, so intranasal spray Dex absorbs faster and feels more comfortable [16,17]. The results of our study showed that intranasal spray Dex $1 \mathrm{ug} / \mathrm{kg}$ was effective for clinical sedation for 3045 minutes. Dex produces an "arousable sedation" or "cooperative sedation" similar to normal sleep: the patient is effectively sedated and easily awakened. Once the arousal stimulus is removed, the patient returns to a sedative state $[18,19]$. In our study, objective sedation was assessed using BIS values while subjective sedation was assessed by the Ramsay sedation score. This study also proves that subjective sedation assessment is consistent with objective sedation assessment. Patients who received Dex intranasally were more sedative with BIS and RSS assessments before anesthesia 
induction compared with the control group. The sedation produced by Dex as patients may be easily aroused and cooperative.

Previous studies have shown that the application of DEX before anesthesia can minimize or eliminate the preoperative anxiety of patients, make them cooperate actively and smoothly through the perioperative period. Dex attenuated the increase in heart rate and blood pressure from precious studies [20]. According to the results of this study, although the SBP and HR decreased in varying degrees, but still in the normal range after the decrease. No significant bradycardia or hypotension was observed. Dex reduces the autonomy of sinoatrial node by selectively acting on myocardial alpha 2 adrenergic receptor, so that no increase in heart rate occurs after stress responses during tracheal intubation and RPP tends to be stable. Compared with the control group, HR, SBP and RPP decreased significantly in group D from before induction of anesthesia to 5 minutes after intubation in the group D. In patients with cardiac risk, decreased heart rate can reduce myocardial oxygen demand and improve coronary artery perfusion, thereby reducing the incidence of perioperative myocardial ischemia [20]. In our study, low-dose Dex intranasally $1 \mathrm{ug} / \mathrm{kg}$ was used to control hemodynamic stress response well.

Cor, E, NE and BS levels were good indicators of stress response in the "alert period". The degree of increase was positively correlated with the intensity of stress stimulation [21]. From precious studies could show that Dex infusion decreased the plasma level of catecholamine and cortisol undergoing Coronary Artery Bypass (CABG) surgery [22]. This supports our findings that significantly lower E and NE in the group D than the control group. If NE, E, Cor, BS can be controlled reasonably and effectively during the perioperative period to alleviate the stress reaction, it is obviously beneficial for patients to pass the perioperative period safely. Because a series of neuroendocrine reactions occur when the body is nervous or anxious before operation, which stimulate the pituitary-adrenal system and activate the sympathetic-adrenal medullary system, and lead to the increase of corticotropinreleasing hormone and catecholamine in blood, NE, E appear in the control group before induction of anesthesia. Cor and BS increased, while group D could inhibit the sympathetic adrenal medulla and pituitary adrenal cortex system, and the plasma concentration of Cor, BS and catecholamine decreased, suggesting that Dex could significantly inhibit the stress reaction induced by preoperative psychological anxiety.

After tracheal intubation, the concentrations of NE, E, Cor and BS in the control group increased significantly, while the concentrations of NE, E, Cor and BS in group D also increased, but were still lower than the baseline values, with no statistical significance $(\mathrm{P}>0.05)$. The results showed that the control group did not inhibit the stress response of endotracheal intubation satisfactorily, and Dex intranasally could significantly inhibit the stress response of the body during endotracheal intubation. The same results have been shown in previous studies. Alpha 2 adrenergic receptor agonists can act on islet B cells, inhibit insulin release, and attenuate the hyperglycemic response induced by sympathetic-adrenal medulla. Interestingly, we found that Dex intranasally did inhibit the hyperglycemic response to tracheal intubation. BS concentration were significantly lower in group D than the control group, and this may reflect attenuation of hyperglycemic response induced by sympathetic-adrenal medulla. Our research has some limitations. although preoperative sedation and attenuate stress response of tracheal intubation were shown using intranasal Dex for sedation in patients undergone gynecological laparoscopic surgery. We still cannot conclude that it is superior to the sedative effect of Dex intravenously. Clinical studies that directly compare the sedative effects of dexmedetomidine intranasal and intravenous injections are needed to assess this.

\section{Conclusion}

Intranasal administration of dexmedetomidine (1ug/ $/ \mathrm{kg})$ 30 min before operation can be effectively applied sedation and alleviate stress response to tracheal intubation, it is as a safe and effective preoperation medication.

\section{Acknowledgement}

Xiaoxia Gu and XiuJuan Tan Co-First Author.

Author Name: Xiaoxia Gu.This author helped Collection of cases, essay writing.

Author Name: XiuJuan Tan.This author helped Collection of cases, essay writing.

Author Name: Yingxia Guo.This author helped Collection of cases, essay writing.

Author Name: Shuyun Cai.This author helped Data collation and analysis.

Author Name:Jinxian Chen.This author helped ELASA test.

Author Name:LiangqingZhang.This author helped Experimental guidance.

\section{References}

1. Lemon J, Edelman S (2007) Psychological adaptation to ICDs and the influence of anxiety sensitivity. Psychol Health Med 12(2): 163-171.

2. Walburn J, Vedhara K, Hankins M (2009) Psychological stress and wound healing in humans: a systematic review and meta-analysis. J Psychosom Res 67(3): 253-271.

3. Martin E, Ramsay G, Mantz J (2003) The role of the alpha 2-adrenoceptor agonist dexmedetomidine in postsurgical sedation in the intensive care unit. Intensive Care Med 18(1): 29-41.

4. Dominic S Carollo, Bobby D Nossaman (2008) Dexmedetomidine: a review of clinical applications. Current Opinion in Anesthesiology 21(4): 457-461.

5. Afonso J, Reis F (2012) Dexmedetomide: current role in anesthesia and intensive care. Rev Bras Anestesiol 62(1): 118-133.

6. Talon MD, Woodson LC, Sherwood ER (2009) Intranasal dexmedetomidine premedication is comparable with midazolam in burn children undergoing reconstructive surgery. J Burn Care Res 30(4): 599-605. 
7. Iirola T, Vilo S, Manner T (2011) Bioavailability of dexmedetomidine after intranasal administration. Eur J Clin Pharmacol 67(8): 825-831.

8. Borland M, Jacobs I, King B (2007) A randomized controlled trial comparing intranasal fentanyl to intravenous morphine for managing acute pain in children in the emergency department. Ann Emerg Med 49(3): 335-340.

9. Lahat E, Goldman M, Barr J, Bistritzer T (2000) Comparison of intranasal mi dazolam with intravenous diazepam for treating febrile seizures in children: pro-spective randomised study BMJ 321(7253): 83-86.

10. Nie Liu Y, Luo Q (2014) Effect of dexmedetomidine combined with sufentanil for post-caesarean section intravenous analgesia: A randomized placebo-controlled study. Obstetric Anesthesia Digest 31: 197.

11. Hwang W, Lee J, Park J, Joo J (2015) Dexmedetomidine versus remifentanil in postoperative pain control after spinal surgery: a randomized controlled study. BMC Anesthesiology 15(1): 1-7.

12. Lu Jianhua, Lu Xin (2016) Effects of two anesthesia methods on immune function of patients undergoing cervical cancer surgery. Chinese Medical Frontier Journal (Electronic Edition) 09-21.

13. Li Ruiyu, Zhang Kangcong, Hu Haoxiang (2015) Effects of dexmedetomidine on heart rate variability in patients undergoing laryngoscopic surgery. Chinese Journal of Endoscopy 21(12): 12621264.

14. Kang Leyan, Liang Jingzhu, Li Binfei (2016) Study on the optimal loading dose of dexmedetomidine for painless fiberoptic bronchoscopy. J Evaluation and analysis of drug use in Chinese hospitals 16(6): 737-739.

ISSN: 2574-1241

DOI: $10.26717 /$ BJSTR.2019.21.003677

Liangqing Zhang. Biomed J Sci \& Tech Res

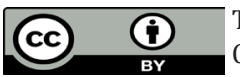

This work is licensed under Creative Commons Attribution 4.0 License

Submission Link: https://biomedres.us/submit-manuscript.php
15. Yuen VM, Irwin MG, Hui TW, Yuen MK, Lee LH (2007) A double blind, crossover assessment of the sedative and analgesic effects of intranasal dexmedetomidine. Anesth Analg 105(2): 374-380.

16. Talon MD, Woodson LC, Sherwood ER (2009) Intranasal dexmedetomidine premedication is comparable with midazolam in burn children undergoing reconstructive surgery. Journal of Burn Care and Research 30: 599-605.

17. VM Yuen, Theresa W Hui, MG Irwin (2010) Optimal timing for the administration of intranasal dexmedetomidine for premedication in children. J Anaesthesia 65: 922-929.

18. Huupponen E, Maksimow A, Lapinlampi P, Meriläinen P, Himanen SL, et al. (2008) Electroencephalogram spindleactivity during dexmedetomidine sedation and physiological sleep. Acta Anaesthesiol Scand 52: 289-294.

19. Mason KP, O Mahony E, Zurakowski D (2009) Effects of dexmedetomidine sedation on the EEG in children. Pediatr Anesth 19: 1175-1183.

20. Okada H, Kurita T, Mochizuki T (2007) The cardio protective effect of dexmedetomidine on global ischaemia in isolated rathearts. Resuscitation 74(3): 538-545.

21.Tsigos C, Chrousos GP (2002) Hypothalamic-pituitary-adrenal axis, neuroendocrine factors and stress. J Psychosom Res 53(4): 865-871.

22. Ahmed M Mukhtar, Eman M Obayah, Amiram, Hassona (2006) The use of deamedetomidine in pediatric cardiac surgery. International Anesthesia research society 103(1): 52-56.

$\begin{array}{ll}\text { BIOMEDICAL } & \text { Assets of Publishing with us } \\ \text { RESEARCHES } & \text { - Global archiving of articles } \\ \text { - Immediate, unrestricted online access } & \text { - Rigorous Peer Review Process } \\ & \text { - Authors Retain Copyrights }\end{array}$

